\title{
Calidad de higiene en salas de cirugía por luminometría de adenosín trifosfato*
}

\section{Quality of Cleaning on Surgery Room by Adenosin Triphosfate Luminometry}

\section{Qualidade de higiene em salas de cirurgia por luminometria de adenosina trifosfato}

Fecha de recepción: 30-05-14 Fecha de aceptación: 22-09-14 Disponible en línea: 01-07-14 doi: 10.11144/Javeriana.rgyps13-27.chsc

Cómo citar este artículo:

Dávila-Ramírez FA, Díaz-Villamil NT, Fajardo-Granados D, Jiménez-Cruz C. Calidad de higiene en salas de cirugía por luminometría de adenosín trifosfato. Rev. Gerenc. Polít. Salud. 2014; 13(27): 266-273. http:// dx.doi.org/10.11144/Javeriana.rgyps13-27.chsc

\author{
Fabián Antonio Dávila-Ramírez** \\ Nancy Teresa Díaz-Villamil*** \\ David Fajardo-Granados**** \\ Camilo Jiménez-Cruz $* * * * *$
}

\footnotetext{
Artículo de investigación, fecha de inicio: 7 de abril del 2014, fecha de finalización: 17 de mayo del 2014. Nombre del departamento y la institución a los que se debe atribuir el trabajo: Universidad Colegio Mayor Nuestra Señora del Rosario y Méderi Hospital Universitario Mayor. No existen conflictos de interés por entidad financiera o patrocinadora en este estudio.

** Coordinador de Epidemiología en la Corporación Hospitalaria Juan Ciudad (Bogotá, Colombia), Méderi Hospital Universitario Mayor, médico epidemiólogo de la Fundación Universitaria Juan N. Corpas. Correspondencia: Dr. Fabián Dávila; correo electrónico: fadavilar@gmail.com. Dirección física: Calle 63B, \# 71C-48 interior 3.

*** Coordinadora de esterilización e instrumentación quirúrgica en la Corporación Hospitalaria Juan Ciudad (Bogotá, Colombia), Méderi Hospital Universitario Mayor, profesional en Instrumentación Quirúrgica, Fundación Universitaria del Área Andina.

***** Estudiante de Medicina de la Escuela de Ciencias de la Salud de la Universidad Colegio Mayor Nuestra Señora del

$266^{* * * * *} \quad \begin{aligned} & \text { Rosario (Bogotá, Colombia). } \\ & \text { Estudiante de Medicina de la Escuela de Ciencias de la Salud de la Universidad Colegio Mayor Nuestra Señora del }\end{aligned}$ Rosario (Bogotá, Colombia).
} 


\section{Resumen}

Objetivo: evaluar la calidad del proceso de desinfección, medido en unidades relativas de luz (URL) en superficies ambientales de salas de cirugía, instrumentos reutilizables y de reuso mediante el equipo luminómetro 3M Clean Trace. Materiales: con un punto de corte en 250 uRL, para definir adecuada calidad de la desinfección, se evaluó una muestra por conveniencia de 39 elementos: seis críticos de reuso, seis superficies ambientales y veintisiete de reutilización de difícil limpieza. Resultados: se encontró mala calidad en la mayoría de elementos evaluados. El gancho monopolar (reuso), la cánula de Frazzier (reutilización) y la mesa de medicamentos (superficie ambiental) fueron los elementos con niveles más elevados en cada grupo. Conclusiones: es necesario mejorar la calidad de limpieza y desinfección en salas de cirugía; el luminómetro demostró ser un instrumento útil en el control de calidad de este proceso; se requieren estudios adicionales para proponer puntos de corte más apropiados.

Palabras clave: adenosín trifosfato; bioluminometría/organización y administración; departamento quirúrgico; desinfección; infección del sitio operatorio

\section{Abstract}

Objective: To assess the quality of the disinfection process, measured in relative light units (URL) on environmental surfaces in operating rooms, reusable and disposable instruments through the luminometer equipment 3M Clean Trace. Materials: With a cut-off point on 250 uRL to define the adequate quality of disinfection, we evaluated a convenience sample of 39 elements, six disposable instruments of critical importance, six environmental surfaces and twenty-seven reusable that are difficult to clean. Results: Bad quality was found on most of the evaluated elements. The monopolar hook (disposable), the Frazzier cannula (reusable) and the medicine counter (environmental surface) were the elements with the highest levels in each group. Conclusions: It is necessary to improve the quality of cleaning and disinfection in operating rooms. The luminometer proved to be a useful instrument in the quality control of this process. Additional studies are required to propose more appropriate cut-off points.

Keywords: adenosine triphosphate; bioluminometry/organization and management; department of surgery; disinfection; infection of the operating site

\section{Resumo}

Objetivo: avaliar a qualidade do processo de desinfeção, medido em unidades relativas de luz (URL), em superfícies ambientais de salas de cirurgia, instrumentos reutilizáveis e de reuso mediante a equipe luminômetro 3M Clean Trace. Materiais: com um ponto de corte em 250 uRL, para definir adequada qualidade da desinfeção, foi avaliada amostra por conveniência de 39 elementos: seis críticos de reuso, sies superfícies ambientais e vinte e sete de reutilização de difícil limpeza. Resultados: Encontrou-se má qualidade na maioria de elementos avaliados. O gancho monopolar (reuso), a cânula de Frazzier (reutilizacao) e a mesa de medicamentos (superfície ambiental) foram os elementos com níveis mais elevados em cada grupo. Conclusões: é preciso melhorar a qualidade de limpa e desinfeção em salas de cirurgia; o luminômetro demonstrou ser instrumento útil no controle de qualidade deste processo; requerem-se estudos adicionais para propor pontos de corte mais apropriados.

Palavras-chave: adenosina trifosfato; bioluminometria/organização e administração; departamento cirúrgico; desinfeção; contaminação do local operatório 


\section{Introducción}

Las infecciones asociadas a la atención en salud (IAAs) que se presentan después de 48 horas, sin evidencia de infección previa, afectaron durante el año 2013 a cerca del 5\% de los pacientes hospitalizados en Estados Unidos $(1,2)$; se reportaron 88000 desenlaces mortales, con un costo aproximado de 4,5 billones de dólares (3).

En países en vía de desarrollo se ha reportado una incidencia acumulada de cinco IAAS por cada cien procedimientos quirúrgicos (2); la mayoría están relacionadas, entre otras causas, con flora bacteriana propia del paciente y el microambiente en los quirófanos (corrientes de aire en la sala de cirugía o contaminación en paredes, piso o instrumental quirúrgico) (4). Es por esto que el seguimiento a las prácticas de limpieza en los centros hospitalarios es considerado un componente importante del control de infecciones (asociadas principalmente a gérmenes como Clostridium difficile, Staphylococcus aureus y Enterococos gram negativos) (5).

Las superficies ambientales susceptibles de contaminación suelen ser clasificadas según la potencial contaminación con sangre y el nivel de desinfección requerido, siendo así clasificadas en: superficies críticas y superficies semicríticas, que entran en contacto con tejido estéril del paciente y con membranas mucosas (respectivamente) y, en consecuencia, requieren desinfección de alto nivel; y superficies no críticas, que entran en contacto con piel indemne. Su bajo riesgo de infección justifica la desinfección de bajo nivel (6).

La forma comúnmente empleada para evaluar la calidad de la desinfección en superficies hospitalarias ha sido siempre la inspección visual, lo cual da un carácter subjetivo a la evaluación del nivel de desinfección. Recientemente se ha desarrollado un método más objetivo que evalúa mediante un equipo llamado luminómetro, el nivel de bioluminiscencia o fotoluminometría de adenosín trifosfato (АTP) en los elementos y superficies desinfectados. El ATP reacciona con el complejo enzimático luciferin-luciderasa, produciendo un nivel de intensidad de luz, por lo que la concentración de ATP en la superficie es directamente proporcional a la intensidad de la luz producida, expresada en unidades relativas de luz (URL). El nivel de bioluminiscencia de ATP corresponde, entonces, a la carga biológica presente en una superficie o elemento, con capacidad de permitir el crecimiento de microorganismos (7).

Algunos estudios han reportado niveles óptimos de limpieza en superficies hospitalarias, con un punto de corte entre 250 y 300 URL (8). Otros han reportado como adecuada la desinfección en materiales de reuso y reutilizables, con niveles inferiores a este punto de corte $(<250$ URL $)$.

Este trabajo pretendió determinar los niveles de URL encontrados en los elementos reutilizables, de reuso y superficies en salas de cirugía del Méderi-Hospital Universitario Mayor (HUM), como estrategia de mejoramiento enmarcada en el sistema obligatorio de garantía de calidad para la desinfección de superficies y elementos en cirugía.

\section{Materiales y métodos}

Se realizó un estudio piloto, observacional, descriptivo para medir la carga biológica por fotoluminometría de ATP en las superficies ambientales, elementos de reutilización y reuso, por medio del luminómetro $3 \mathrm{M}$ Clean Trace. Como punto de corte para establecer una apropiada limpieza y desinfección, se determinaron los niveles menores a 250 URL (8). 
Los elementos para muestreo fueron seleccionados por su uso en procedimientos asociados a infección del sitio operatorio (reportados en el comité normativo de infecciones del Méderi-Hospital Universitario Mayor).

Se tomó una muestra por conveniencia $(\mathrm{n}=39)$ de:

- Seis dispositivos críticos de reuso, llevados a lavado manual. Se escogieron: máscara con bolsa auto expandible (Ambú), gancho monopolar, lápiz electro-bisturí, pinza disectora de 5 $\mathrm{mm}$, tijera laparoscópica y un trocar de $10 \mathrm{~mm}$.

- Veintisiete elementos de reutilización de difícil limpieza (definidos así por la presencia de ranuras, bisagras y elementos canulados). Un elemento de cada pareja se llevó a lavado manual y el otro a máquina de ultrasonido en cada caso, excepto la cureta de Novac, la cánula de Frazzier y la cánula de succiónirrigación. Se escogieron: una cureta de Novac; una cánula de succión; dos clamp vascular; dos cuchilla para hueso; dos jacobs; dos medidores de profundidad 3,5; dos medidores de profundidad 4,5; dos portaagujas; dos pinzas Babcock; dos pinzas Kelly; dos pinzas Kerrison; dos raspa; dos jaritrack; dos tijeras y cánulas de Frazzier.

- Seis superficies ambientales de una sala del servicio de cirugía general. Se evaluaron las siguientes áreas, posteriores al proceso de desinfección: colchoneta quirúrgica, lámpara, mesa de medicamentos, mesa de riñón, monitor de anestesia y perilla de anestesia.

Las variables cuantitativas fueron presentadas con medias y desviación estándar, las variables cualitativas con frecuencias absolutas y relativas. Los resultados se presentaron en tablas de distribución de frecuencia y graficas de dipersión. Asimismo, fueron exportados del equipo de luminometría a una hoja de cálculo de Microsoft Excel, se organizaron en una matriz de datos apilados y se importaron para su análisis al software estadístico SPSS 22.0.

\section{Resultados}

Se encontraron niveles elevados de URL en la mayoría de los elementos evaluados; el gancho monopolar (reuso-critico), la cánula de Frazzier vascular (reutilización-crítico) y la mesa de medicamentos (superficie ambientalno crítico) fueron los elementos con valores más elevados en cada grupo (ver tabla 1 ).

TABLA 1. NIVELES DE URL POR TIPO ELEMENTO E HIGIENE REALIZADA

\begin{tabular}{|c|c|c|c|c|c|c|c|}
\hline \multirow{2}{*}{ Tipo de elemento } & \multirow{2}{*}{$\begin{array}{l}\text { Caracterís- } \\
\text { tica }\end{array}$} & \multirow{2}{*}{$\begin{array}{l}\text { Criti- } \\
\text { cidad }\end{array}$} & \multirow{2}{*}{$\begin{array}{l}\text { Número } \\
\text { de ele- } \\
\text { mentos }\end{array}$} & \multirow{2}{*}{$\begin{array}{l}\text { Por- } \\
\text { cen- } \\
\text { taje }\end{array}$} & \multicolumn{2}{|c|}{ Niveles de URL en higiene } & \multirow{2}{*}{$\begin{array}{c}\text { Total } \\
\text { general }\end{array}$} \\
\hline & & & & & Manual & Ultrasonido & \\
\hline $\begin{array}{l}\text { R1S Máscara con bolsa auto } \\
\text { expandible }\left(\mathrm{Ambu}^{\circledast}\right)\end{array}$ & Reuso & $\begin{array}{l}\text { No } \\
\text { crítico }\end{array}$ & 1 & $3,7 \%$ & 125 & 0 & 125 \\
\hline R1S Gancho Monopolar & Reuso & Crítico & 1 & $3,7 \%$ & 18177 & 0 & 18177 \\
\hline R1S Lápiz Electro-bisturí & Reuso & Crítico & 1 & $3,7 \%$ & 184 & 0 & 184 \\
\hline R1S Pz Disectora de $5 \mathrm{~mm}$ & Reuso & Crítico & 1 & $3,7 \%$ & 2398 & 0 & 2398 \\
\hline R1S Tijera Laparoscópica & Reuso & Crítico & 1 & $3,7 \%$ & 1769 & 0 & 1769 \\
\hline R1S Trocar & Reuso & Crítico & 1 & $3,7 \%$ & 385 & 0 & 385 \\
\hline 1Q2A Cureta Novac & Reutilización & Crítico & 1 & $3,7 \%$ & 0 & 125 & 125 \\
\hline
\end{tabular}




\begin{tabular}{|c|c|c|c|c|c|c|c|}
\hline \multirow{2}{*}{ Tipo de elemento } & \multirow{2}{*}{$\begin{array}{l}\text { Caracterís- } \\
\text { tica }\end{array}$} & \multirow{2}{*}{$\begin{array}{l}\text { Criti- } \\
\text { cidad }\end{array}$} & \multirow{2}{*}{$\begin{array}{l}\text { Número } \\
\text { de ele- } \\
\text { mentos }\end{array}$} & \multirow{2}{*}{$\begin{array}{l}\text { Por- } \\
\text { cen- } \\
\text { taje }\end{array}$} & \multicolumn{2}{|c|}{ Niveles de URL en higiene } & \multirow{2}{*}{$\begin{array}{c}\text { Total } \\
\text { general }\end{array}$} \\
\hline & & & & & Manual & Ultrasonido & \\
\hline IQ1S Clamp vascular & Reutilización & Crítico & 2 & $7,4 \%$ & 1354 & 19731 & 21085 \\
\hline IQ1S Cuchilla para hueso & Reutilización & Crítico & 2 & $7,4 \%$ & 3578 & 240 & 3818 \\
\hline IQ1S Jacobs & Reutilización & Crítico & 2 & $7,4 \%$ & 2805 & 1037 & 3842 \\
\hline IQ1S Medidor profundidad 3,5 & Reutilización & Crítico & 2 & $7,4 \%$ & 859 & 1661 & 2520 \\
\hline IQ1S Medidor profundidad 4,5 & Reutilización & Crítico & 2 & $7,4 \%$ & 720 & 875 & 1595 \\
\hline IQ1S Portaagujas & Reutilización & Crítico & 2 & $7,4 \%$ & 28 & 188 & 216 \\
\hline IQ1S Pz Babcock & Reutilización & Crítico & 2 & $7,4 \%$ & 103 & 73 & 176 \\
\hline IQ1S Pz Kelly & Reutilización & Crítico & 2 & $7,4 \%$ & 412 & 76 & 488 \\
\hline IQ1S Pz Kerrison & Reutilización & Crítico & 2 & $7,4 \%$ & 14 & 111 & 125 \\
\hline IQ1S Raspa & Reutilización & Crítico & 2 & $7,4 \%$ & 66 & 106 & 172 \\
\hline IQ1S Sep Jaritrak & Reutilización & Crítico & 2 & $7,4 \%$ & 815 & 530 & 1345 \\
\hline IQ1S Tijeras & Reutilización & Crítico & 2 & $7,4 \%$ & 675 & 410 & 1085 \\
\hline IQ2A Cánula de Frazzier & Reutilización & Crítico & 1 & $3,7 \%$ & 0 & 21001 & 21001 \\
\hline IQ2A Cánula succión-irrigación & Reutilización & Crítico & 1 & $3,7 \%$ & 0 & 3 & 3 \\
\hline Colchoneta & $\begin{array}{l}\text { Superficie } \\
\text { quirúrgica }\end{array}$ & $\begin{array}{c}\text { No } \\
\text { crítico }\end{array}$ & 1 & $3,7 \%$ & 729 & 0 & 729 \\
\hline Lámpara & $\begin{array}{l}\text { Superficie } \\
\text { quirúrgica }\end{array}$ & $\begin{array}{c}\text { No } \\
\text { crítico }\end{array}$ & 1 & $3,7 \%$ & 8198 & 0 & 8198 \\
\hline Mesa de medicamentos & $\begin{array}{l}\text { Superficie } \\
\text { quirúrgica }\end{array}$ & $\begin{array}{c}\text { No } \\
\text { crítico }\end{array}$ & 1 & $3,7 \%$ & 10743 & 0 & 10744 \\
\hline Mesa de riñón & $\begin{array}{l}\text { Superficie } \\
\text { quirúrgica }\end{array}$ & $\begin{array}{c}\text { No } \\
\text { crítico }\end{array}$ & 1 & $3,7 \%$ & 1723 & 0 & 1723 \\
\hline Monitor de anestesia & $\begin{array}{l}\text { Superficie } \\
\text { quirúrgica }\end{array}$ & $\begin{array}{c}\text { No } \\
\text { crítico }\end{array}$ & 1 & $3,7 \%$ & 1239 & 0 & 1239 \\
\hline Perilla de anestesia & $\begin{array}{l}\text { Superficie } \\
\text { quirúrgica }\end{array}$ & $\begin{array}{c}\text { No } \\
\text { crítico }\end{array}$ & 1 & $3,7 \%$ & 2648 & 0 & 2648 \\
\hline
\end{tabular}

Los elementos: máscara con bolsa auto expandible (Ambu), lápiz electro-bisturí, trocar de $10 \mathrm{~mm}$, cureta de Novac, portaagujas, pinzas Babcok, pinzas Kerrison, raspa y la cánula de succión irrigación fueron los que presentaron mejores niveles de desinfección (ver tabla 1).

Los niveles de URL posteriormente a la higiene manual y ultrasonido, no mostraron dife- rencias significativas por nivel de criticidad de los elementos (el test de Levene sugiere no igualdad entre varianza de los niveles de URL en elementos críticos vs. elementos no críticos) (ver figuras 1 y 2).

La diferencia de promedios y desviaciones estándar para la limpieza manual y por ultrasonido no fue significativa (ver tabla 2).

TABLA 2. DifERENCIA DE PROMEdIOS Y DESVIACIONES EN NIVELES DE URL DE HIGIENE MANUAL Y ULTRASONIDO PARA ELEMENTOS CRÍTICOS Y NO CRÍTICOS

\begin{tabular}{|l|l|l|l|l|l|}
\hline $\begin{array}{l}\text { Niveles de uRL en } \\
\text { higiene }\end{array}$ & $\begin{array}{l}\text { Diferencia de } \\
\text { medias }\end{array}$ & $\begin{array}{l}\text { Diferencia de } \\
\text { error estándar }\end{array}$ & \multicolumn{2}{|l|}{$\begin{array}{l}\text { Intervalo de confi- } \\
\text { anza del 95\% }\end{array}$} & $\begin{array}{l}\text { Valor de p: Sig. } \\
\text { (bilateral) }\end{array}$ \\
\hline Manual & 1912,19 & 1774,19 & $-1741,83$ & 0,29 & 5566,2 \\
\hline Ultrasonido & $-2308,35$ & 2371,4 & $-7192,34$ & 0,34 & 2575,64 \\
\hline
\end{tabular}




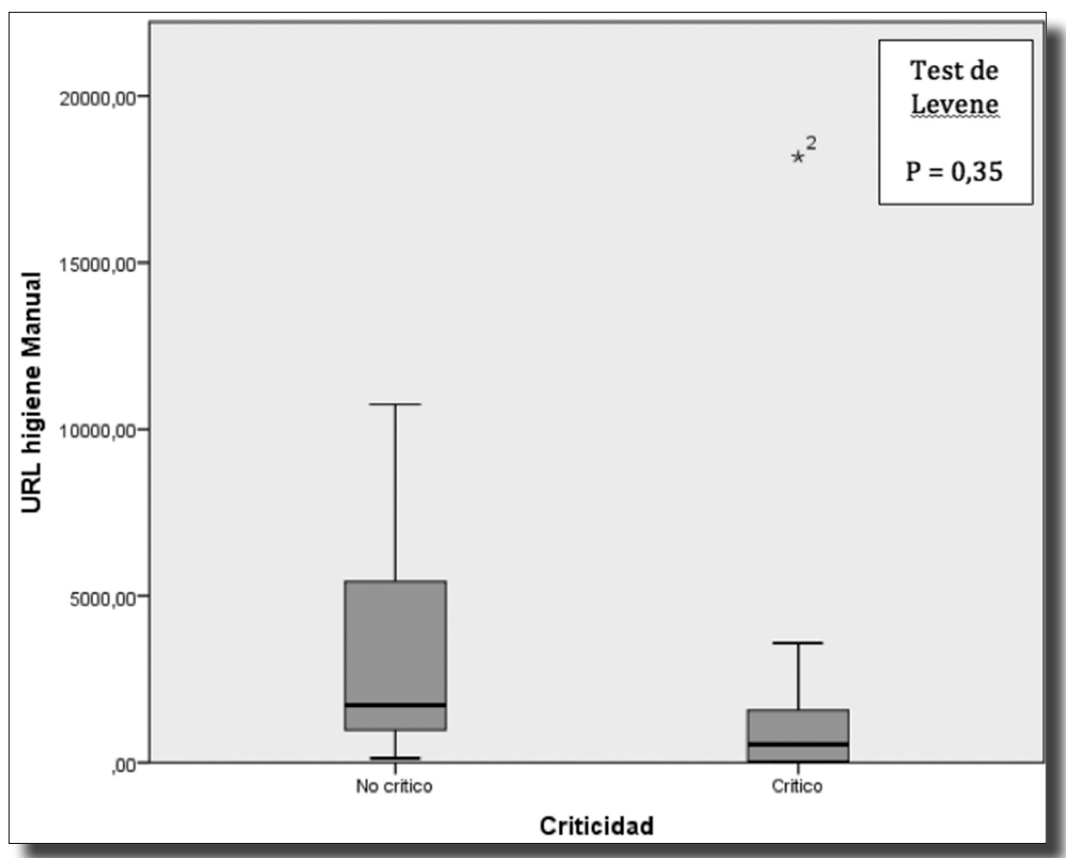

Figura 1. Diagrama de Cajas (box Plot) Para niveles de URL En higiene Manual SEgún CRiticidad DEL ELEMENTO

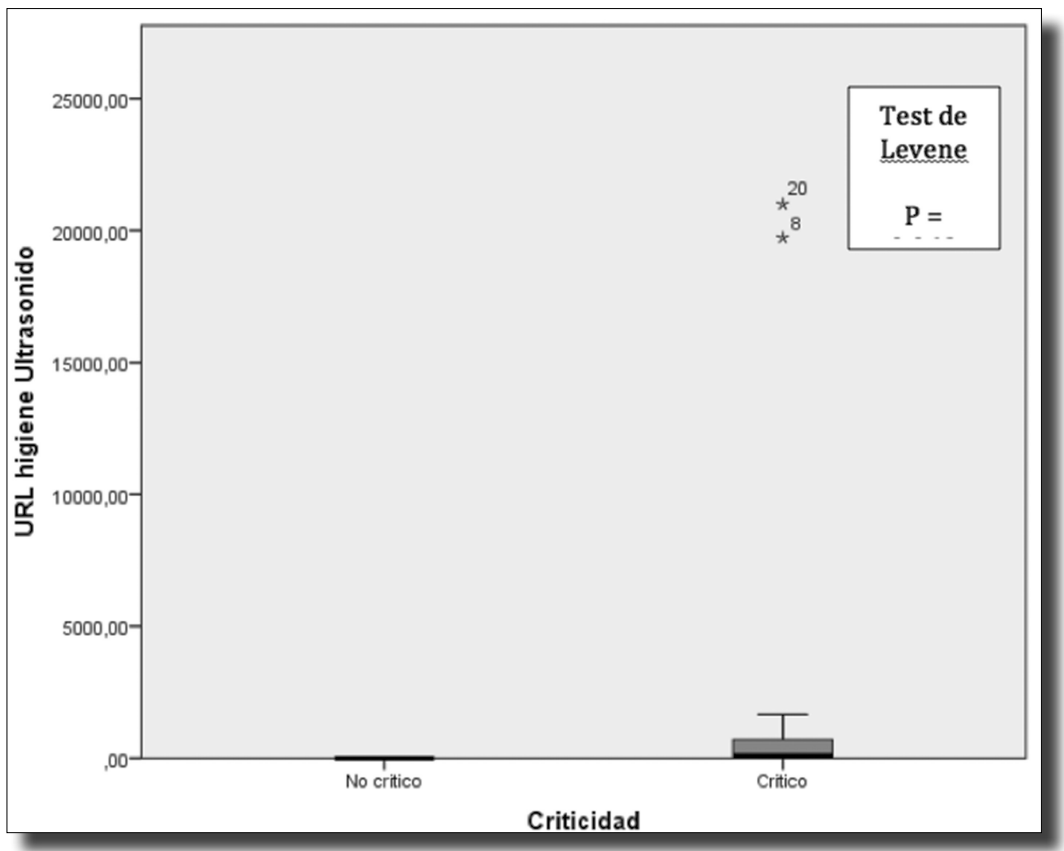

Figura 2. Diagrama de CAJas ( CRITICIDAD DEL ELEMENTO 


\section{Discusión}

El presente estudio evaluó - por sus implicaciones en el riesgo de infección en el paciente- la calidad de la desinfección de los materiales quirúrgicos reutilizables, de reuso y superficies ambientales en los quirófanos en Méderi-Hospital Universitario Mayor, mediante el método de bioluminiscencia por ATP con luminometría.

A la fecha, durante la revisión de la bibliografía (realizada en literatura indexada dei: Pubmedy Scholar Google) no encontramos estudios que evaluaran la calidad de la desinfección en salas de cirugía mediante esta técnica.

En nuestra institución, después de cada procedimiento, las superficies ambientales se limpian por métodos manuales; los elementos reutilizables y reuso son llevados (según las características del dispositivo) a lavado manual o en máquina de ultrasonido (por ejemplo, los dispositivos canulados o fenestrados son llevados a ultrasonido).

La limpieza de superficies ambientales quirúrgicas se consideró de baja calidad en todas las muestras evaluadas.

Las limitaciones del presente estudio se corresponden con las descritas para estudios observacionales, que incluyen: subjetividad personal (generar sesgos de medición y reporte), la falta de estudios previos en elementos de reutilización y reusos impide hacer comparaciones.

Los puntos de corte de URL escogidos se basaron en estudios previos en áreas hospitalarias no quirúrgicas $(7,8)$; la comprobación de las hipótesis de mala calidad en la limpieza y desinfección requiere estudios analíticos y validación de puntos de corte que permitan definir niveles de protección del riesgo de infección del sitio operatorio.
No se apreciaron diferencias entre el lavado manual y la limpieza por ultrasonido en los elementos evaluados.

Como fortaleza, este estudio corresponde a la primera descripción de un procedimiento objetivo para el análisis cuantitativo del proceso de desinfección en el espacio quirúrgico. Se expone la necesidad de estandarizar la desinfección de los elementos quirúrgicos (en especial los elementos de reuso) como pilar de la calidad de la desinfección que permita mitigar el riesgo de infección del sitio operatorio.

Los resultados del presente estudio serán de utilidad para estandarizar la vigilancia y el control de calidad en el proceso de desinfección

La coordinación de esfuerzos entre el Comité de Infecciones, Gestión Ambiental y la Central de Esterilización del Méderi-Hospital Universitario Mayor en la capacitación de programas reinducción del personal y la verificación del proceso de limpieza y desinfección en salas de cirugía, así como del instrumental quirúrgico, resultó ser una estrategia efectiva en el control de infecciones de sitio operatorio, mediante la supervisión, el seguimiento y el registro de control con la aplicación del luminómetro en los dispositivos y superficies escogidos en nuestro estudio.

\section{Conclusiones}

Es necesario estandarizar el procedimiento de limpieza en superficies ambientales y en elementos de reuso y de reutilización de salas de cirugía.

El luminómetro es un instrumento que permite la evaluación objetiva del control de calidad en el proceso de desinfección en salas de cirugía. 
Se requieren estudios adicionales de cohorte y prospectivos, para proponer un punto de corte de URL adecuado en estos elementos.

\section{Conflicto de interés}

Los autores no presentaron ningún conflicto de interés para el desarrollo del presente trabajo. Los elementos para toma de muestras fueron financiados con recursos propios de la institución. No existió ninguna financiación de industrias privadas como $3 \mathrm{M}$ y Ambu, mencionadas en el estudio.

\section{Referencias bibliográficas}

1. Ávila-Figueroa C, Cashat-Cruz M, Aranda-Patrón E, León AR, Justiniani N, Pérez-ricárdez L et ál. Prevalencia de infecciones nosocomiales en niños: encuesta de 21 hospitales en México. Salud Pública Mex. 1999; 41 (S1).

2. Allegranzi B, Bagheri Nejad S, Combescure C, Graafmans W, Attar H, Donaldson L et ál. Burden of Endemic Health-Care-Associated Infection in Developing Countries: Systematic Review and Meta-Analysis. Lancet [Internet], 2011 Jan. 15; 377 (9761): 228-41 [acceso: 24 de marzo del 2014].

3. Petroudi D. Nosocomial Infections and Staff Hygiene. J Infect Dev Ctries [Internet]. 2009 Mar. 1; 3 (02): 152-6 [acceso: 7 de abril del 2014].

4. Ayliffe GA. Role of the Environment of the Operating Suite in Surgical Wound Infection. Rev Infect Dis [Internet]; 13 (Suppl.) 1: S 800-4 [acceso: 7 de abril del 2014].

5. Donskey CJ. Does Improving Surface Cleaning and Disinfection Reduce Health Care-Associated Infections? Am J Infect Control [Internet]. 2013 May. 1; 41 (5 Suppl): S12-9 [acceso: 10 de abril del 2014].

6. Rutala WA, Weber DJ. Disinfection and Sterilization: An Overview. Am J Infect Control [Internet]. 2013 May. 1; 41 (5 Suppl): S2-5 [acceso: $1^{\text {o }}$ de abril del 2014].

7. Costa PD, Andrade NJ, Passos FJV, Brandão SCC, Rodrigues CGF. ATP - Bioluminescence as a Technique to Evaluate the Microbiological Quality of Water in Food Industry. Brazilian Arch Biol Technol. Tecpar [Internet]. 2004 Jul.; 47 (3): 399-405 [acceso: 8 de abril del 2014].

8. Boyce JM, Havill NL, Dumigan DG, Golebiewski M, Balogun O, Rizvani R. Monitoring the Effectiveness of Hospital Cleaning Practices by Use of an Adenosine Triphosphate Bioluminescence Assay. Infect Control Hosp Epidemiol [Internet]. 2009 Jul. 2; 30 (7): 678-84 [acceso: 8 de abril del 2014]. 